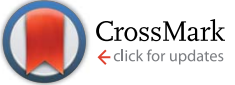

Cite this: RSC Adv., 2016, 6, 57820
Received 10th May 2016 Accepted 8th June 2016

DOI: 10.1039/c6ra12194b

www.rsc.org/advances

\section{Catalyst-free site-specific surface modifications of nanocrystalline diamond films via microchannel cantilever spotting $\dagger$}

\author{
Marina Davydova, ${ }^{\text {a }}$ Andres de los Santos Pereira, ${ }^{\mathrm{b}}$ Michael Bruns, ${ }^{\mathrm{c}}$ \\ Alexander Kromka, ${ }^{a}$ Egor Ukraintsev, ${ }^{a}$ Michael Hirtz ${ }^{d}$ and Cesar Rodriguez- \\ Emmenegger*be
}

\begin{abstract}
The properties of nanocrystalline diamond (NCD) films offer great potential for the creation of various sensing and photonic devices. A great challenge in order to materialize such applications lies in achieving the micrometrically resolved functionalization of NCD surfaces. In the present work, we introduce a facile approach to meet this challenge employing the novel strain-promoted alkyne-azide cycloaddition "click" chemistry reaction, a catalyst-free ligation protocol compatible with biomolecules. The ability to achieve well-resolved multicomponent patterns with high reproducibility is demonstrated, paving the way for the fabrication of novel devices based on micropatterned NCD films.
\end{abstract}

\section{Introduction}

Besides being the hardest of all materials, diamond occupies a special place in industry. Its electronic properties, including a $5.47 \mathrm{eV}$ band gap, make diamond a promising semiconductor material. Currently, diamond can be prepared synthetically either as a bulk material under high pressure and high temperature or in the form of thin films by chemical vapor deposition (CVD). The surface electronic properties of intrinsic diamond can be significantly altered by contact with oxidative or reductive plasma. Diamond hydrogen or oxygen surface termination results in marked differences in properties such as electrical conductivity, electron affinity, and surface wettability. ${ }^{1}$ Bulk doping with boron (p-type) and nitrogen or phosphorous (n-type) can also be incorporated during the thin film deposition process to tune the semiconductor properties. ${ }^{2,3}$

Microwave CVD has become the preferred deposition technique for opto-electronic applications due to its advantages,

${ }^{a}$ Institute of Physics v.v.i., Academy of Sciences of the Czech Republic, Cukrovarnicka 10, 16200 Prague 6, Czech Republic. E-mail: davydova@fzu.cz

${ }^{b}$ Department of Chemistry and Physics of Surfaces and Biointerfaces, Institute of Macromolecular Chemistry v.v.i., Academy of Sciences of the Czech Republic, Heyrovsky Sq. 2, 16206 Prague 6, Czech Republic

'Institute for Applied Materials (IAM), Karlsruhe Nano Micro Facility (KNMF), Karlsruhe Institute of Technology (KIT), 76344, Eggenstein-Leopoldshafen, Germany ${ }^{d}$ Institute of Nanotechnology (INT), Karlsruhe Nano Micro Facility (KNMF), Karlsruhe Institute of Technology (KIT), 76344, Eggenstein-Leopoldshafen, Germany

${ }^{e} D W I$ - Leibniz Institute for Interactive Materials and Institute of Technical and Macromolecular Chemistry, RWTH Aachen University, Forckenbeckstraße 50, 52074 Aachen, Germany.E-mail: rodriguez@dwi.rwth-aachen.de

$\dagger$ Electronic supplementary information (ESI) available. See DOI: 10.1039/c6ra12194b including large area deposition, high growth rate, and high surface quality. This technique usually yields films composed of nanocrystals, termed nanocrystalline diamond (NCD). Their inexpensive production on a large variety of substrates of arbitrary size and shape compared to monocrystalline diamond makes NCD films a more feasible alternative for industrial applications. ${ }^{\mathbf{4}, 5}$

Recently, NCD films have been utilized for advanced electronic devices in the field of sensing, including gas sensors and photonic devices, as well as biosensors. ${ }^{6-9}$ As an example, hydrogen-terminated NCD films have been shown to exhibit changes in their surface conductivity in the presence of phosgene and could be utilized as a physical transducer to detect this gas. ${ }^{10}$ In a similar fashion, the amperometric detection of glucose was accomplished by the immobilization of glucose oxidase enzyme on NCD films. ${ }^{11}$

Generally, diamond-based sensing devices rely on the binding and reaction of molecules directly at the NCD surface. ${ }^{12,13}$ In the vast majority of cases, the specificity of the sensor response depends on the presence of appropriate receptors immobilized onto the surface. ${ }^{9}$ Thus, in order to extend the applications of NCD films in the field of sensing, as well as biotechnology, the ability to functionalize their surface is a prerequisite. ${ }^{9}$ In particular, being able to encode different functionalities in precise regions of NCD films can be envisioned as a feasible route for the design of surfaces with controlled interactions in biological environments, ${ }^{14,15}$ as well as high-throughput sensors.

Surface immobilization of bioactive molecules by spotting and printing methods has drawn much attention in recent years, as it is a base technique for the preparation of structured, bioactive surfaces. ${ }^{16-19}$ For highest feature resolution in the 
nanoscale, techniques like dip-pen nanolithography (DPN) and polymer pen lithography (PPL) offer interesting lithography routes. ${ }^{\mathbf{1 6 , 2 0}}$ While ink jet printing offers contactless highthroughput spotting at larger length scales (usually $>50 \mu \mathrm{m}$ ), techniques as microcontact printing $(\mu \mathrm{CP})$ and spotting with microchannel cantilevers $(\mu \mathrm{CS})^{21}$ can readily address the scale range down to a few microns. ${ }^{18,22}$ The deposition of microreactors on a surface to allow liquid phase coupling chemistry to take place for prolonged time or transfer of otherwise not writable species is used in DPN and PPL in the form of matrixassisted transfer (MA-DPN/MA-PPL). ${ }^{23}$ Similarly, $\mu \mathrm{CS}$ was previously demonstrated for the spotting of femtoliter-scale reaction chambers for surface immobilization of bioactive substances into microarrays by "click" chemistry. ${ }^{24,25}$ The click reaction chosen in the previously mentioned approaches is the copper-catalyzed alkyne-azide cycloaddition (CuAAC), that has proven enormously useful for biochemical coupling reactions since the introduction of the concept by Sharpless. ${ }^{26,27}$ In particular, several examples have been reported in which the CuAAC reaction was employed to functionalize the surface of diamond materials, including nanoparticles, ${ }^{28}$ detonation diamond nanoparticles, ${ }^{29}$ or conductive diamond, ${ }^{30-34}$ which provided an avenue to electrochemically stable, functional electrodes.

However, since terminal alkynes are rather unreactive towards azides, the efficiency of the CuAAC reaction strictly relies on the presence of a $\mathrm{Cu}(\mathrm{I})$ catalyst species, often achieved by combining $\mathrm{CuSO}_{4}$ with sodium ascorbate. This requirement poses a serious drawback for several applications. ${ }^{35}$ In the electronic fields the presence of $\mathrm{Cu}$-ions on the surface can disrupt monolayer conductivity. ${ }^{36}$ Particularly in many applications in the biological field, the presence of such ions cannot be tolerated, as they are cytotoxic and can disrupt the conformation of the biomacromolecules to be patterned, including proteins and DNA. ${ }^{37,38}$ Though extensive washing or the use of heterogeneous catalysis can often alleviate the problem, concerns about the need for $\mathrm{Cu}$-ions remain. ${ }^{39}$

In order to overcome these limitations altogether, reactions are sought which proceed rapidly at room temperature, in aqueous solvents, and do not require any catalyst. In this regard the copper-free strain-promoted alkyne-azide cycloaddition (SPAAC) click reaction, developed by Bertozzi and coworkers, has attracted considerable attention. ${ }^{35,40}$ It relies on the use of strained cyclooctynes and their derivatives to activate the reactions. Thus, reaction rates are very fast without the need for a metal catalyst. Its bioorthogonality and compatibility with biological molecules has been thoroughly demonstrated, as the reaction could even be applied to in vivo labeling and imaging. ${ }^{40,41}$

Importantly, SPAAC reactions have been found to be suitable for the functionalization of material surfaces such as silicon, gold, or glass but also quantum dots, nanoparticles and polymer nanofibers. ${ }^{42,43}$ Silane chemistry was used by Popik and coworkers to mediate the covalent surface attachment of the strained alkyne to glass slides, after which the SPAAC reaction was carried out in a surface-confined fashion. ${ }^{\mathbf{4 4}}$ A further refinement of this strategy consisted in the surface grafting of functionalizable polymer brushes and their side-chain substitution with "masked" dibenzocyclooctynes (DBCO), which could be converted to the strained alkyne form by irradiation with UV-light, allowing the ensuing SPAAC reaction to take place in a spatially resolved manner. ${ }^{45}$ A later approach used microcontact printing in order to achieve the patterning. ${ }^{46}$ Postpolymerization-functionalized surface-grafted polymer brushes were also used to compare the reaction kinetics of two types of DBCO-derivatives with an azide. ${ }^{47}$

On the other hand, the surface immobilization of azide groups followed by SPAAC reaction with a cyclic alkyne present in solution has also been exploited. ${ }^{48}$ Various surface functionalization strategies based on SPAAC have been reviewed recently. ${ }^{49}$ The advantages of this novel catalyst-free click reaction can be therefore envisioned to further expand the power of $\mu \mathrm{CS}$, PPL, and DPN.

Herein, we demonstrate a facile route for the surface functionalization of NCD films via $\mu \mathrm{CS}$ in order to create small-scale patterns suitable for different types of sensor applications. For this purpose, we create functionalized silane coatings bearing chemical groups that can participate in either the established CuAAC reaction or the catalyst-free SPAAC reaction. Moreover, we compare the effectiveness of both protocols for $\mu \mathrm{CS}$. We further assess the possibility of employing this method to create well-resolved multi-component patterns on the NCD film surface.

\section{Experimental}

\subsection{Materials}

(3-Glycidyloxypropyl)trimethoxysilane (98\%), dibenzocyclooctyneamine (DBCO), and propargylamine were purchased from SigmaAldrich (Czech Republic). Silicon wafers used as the substrate for nanocrystalline diamond film deposition were acquired from Siegert Wafer GmbH (Aachen, Germany). The fluorescent inks tetramethylrhodamine (TAMRA) azide, Alexa fluor 488-azide, Cy5azide, $\mathrm{CuSO}_{4}$ and ascorbic acid were acquired from Sigma-Aldrich (Germany). (3-Glycidyloxypropyl)trimethoxysilane was purified by vacuum distillation.

\subsection{Methods}

\subsubsection{Surface modification}

2.2.1.1 Deposition of nanocrystalline diamond films. The deposition of nanocrystalline diamond film on the silicon substrates proceeded in two steps: (i) seeding for $40 \mathrm{~min}$, and (ii) microwave plasma-enhanced chemical vapor deposition (PECVD). Prior to the diamond deposition, the substrates were ultrasonically cleaned in isopropyl alcohol and afterwards rinsed with deionized water. Then, the substrates were immersed for $40 \mathrm{~min}$ into an ultrasonic bath containing an ultra-dispersed diamond colloidal suspension. This process leads to the formation of a 5 to $25 \mathrm{~nm}$ thin layer of nanodiamond powder on the Si substrate. The nucleation procedure was followed by the microwave PECVD using a pulsed-linear antenna microwave chemical plasma system (Roth\&Rau AK 400). ${ }^{4}$ The deposition of the NCD layer was carried out using 
a microwave power of $2 \times 1700 \mathrm{~W}$, pressure of $0.1 \mathrm{mbar}$, with a gas mixture of $\mathrm{H}_{2}, \mathrm{CH}_{4}$ and $\mathrm{CO}_{2}(100 / 5 / 30 \mathrm{sccm})$ and substrate temperature of $500{ }^{\circ} \mathrm{C}$.

2.2.1.2 Self-assembled monolayer (SAM) of epoxide. The substrates coated with a deposited NCD layer were rinsed twice with ethanol and deionized water, dried by blowing with nitrogen, and exposed to air plasma for $20 \mathrm{~min}$. Subsequently they were immersed in a $1 \%(\mathrm{v} / \mathrm{v})$ solution for (3-glycidyloxypropyl)trimethoxysilane in dry toluene and kept for $8 \mathrm{~h}$ at room temperature in a dry environment. After this time, the samples were removed from the solution, rinsed with toluene and acetone and dried by blowing with nitrogen.

2.2.1.3 Immobilization of the clickable group. Immediately after silanization, the samples were immersed in a solution of either propargylamine $2 \%(\mathrm{v} / \mathrm{v})$ or dibenzocyclooctyne-amine (DBCO, $1 \mathrm{mg} \mathrm{mL}^{-1}$ ) in dry dichloromethane in individual sealed vials. The reaction was allowed to proceed at $35{ }^{\circ} \mathrm{C}$ for 12 h. Subsequently, the samples were rinsed with dichloromethane, acetone, ethanol, and water, and dried by blowing with nitrogen.

\subsubsection{Pattern fabrication}

2.2.2.1 Dye solutions used for patterning. All fluorescent dye solutions used in this work in the $\mu \mathrm{CS}$ procedure ("inks") consisted of either TAMRA-azide, Alexa fluor 488-azide, or Cy5azide, in all cases at a concentration of $100 \mu \mathrm{g} \mathrm{mL}{ }^{-1}$, dissolved in a mixture of water/glycerol ( $7: 3)$ to prevent drying. For the solutions to be patterned on propargyl-functional NCD films via $\mathrm{CuAAC}, \mathrm{CuSO}_{4}(10 \mathrm{mM})$ and sodium ascorbate (20 $\mathrm{mM}$ ) were added as catalyst. In the case of the dye solutions patterned on DBCO-functional NCD films using the SPAAC reaction, no catalyst was added.

2.2.2.2 Pattern writing via $\mu C S$. All patterns were written with an NLP 2000 system (NanoInk, USA) using on-board software (NanoInk, USA) that allows programming of the dwell time and position of the surface patterning tool (SPT-S-C10S, Bioforce Nanosciences). Prior to use, the SPT pens were plasma cleaned by oxygen plasma (10 sccm $\mathrm{O}_{2}, 100 \mathrm{mTorr}, 30 \mathrm{~W}$ for 5 $\mathrm{min})$. Then, the SPT was mounted onto the tip holder by doublesided sticky tape and the pen reservoir was filled with $1 \mu \mathrm{L}$ of the corresponding dye solution. The spotting proceeded at a relative humidity of $60 \%$ and with the sample stage titled by $8^{\circ}$ with respect to the tip. For all patterns, a dwell time of $0.5 \mathrm{~s}$ was used. After the lithography process, the click reaction between surface and azide-functional dye (either CuAAC or SPAAC) was allowed to proceed for $1 \mathrm{~h}$ in dark at a constant humidity of $60 \%$. Subsequently, the samples were rinsed with ethanol and deionized water to remove the excess solution, and dried by blowing with nitrogen.

2.2.3 Characterization methods. Surface characterization was performed on pristine (as-grown) and chemically modified NCD films.

2.2.3.1 Scanning electron microscopy (SEM). The morphology of the nanocrystalline diamond films was obtained by scanning electron microscopy (SEM, Raith e_LiNE). The images were taken at accelerating voltages of the primary electrons of $10 \mathrm{kV}$ and in-lens detector was used.
2.2.3.2 Fluorescence microscopy. Fluorescence microscopy images were taken with a Nikon upright fluorescence microscope (Eclipse 80i), equipped with a sensitive camera CoolSNAP $\mathrm{HQ}^{2}$ (Photometrics). The broadband excitation light source (Intensilight illumination) is combined with sets of filters (Texas Red, FITC, DAPI) to separate excitation and emission spectra, depending on the used dye molecule. The fluorescence image analysis for determination of spot area was done in ImageJ software by applying a threshold to the raw images and using the particle analysis tool. ${ }^{\mathbf{5 0}}$

2.2.3.3 Atomic force microscopy (AFM). The surface topography of the NCD films was characterized in quantitative nanomechanical mapping mode of PeakForce AFM system (ICON, Bruker) using new Multi75AL cantilever treated in $\mathrm{CF}_{4}$ plasma (pressure $100 \mathrm{mTorr}$ ) for $30 \mathrm{~s}$. The root-mean-square roughness $\left(R_{\mathrm{RMS}}\right)$ was evaluated from two scan area sizes: $5 \times$ $5 \mu \mathrm{m}^{2}$ and $1 \times 1 \mu \mathrm{m}^{2}$.

2.2.3.4 Raman spectroscopy. Micro-Raman spectroscopy (InVia Reflex by Renishaw) using a HeCd laser with a $442 \mathrm{~nm}$ excitation wavelength, and spot diameter $2 \mu \mathrm{m}$ was employed to assess the allotropic components of the NCD films.

2.2.3.5 Thickness measurement. The thickness of the NCD films was evaluated from the interference fringes of the reflectance spectra measured in UV-vis-NIR region using a custommade device and commercial software for modelling the optical properties of the thin film.

2.2.3.6 X-ray photoelectron spectroscopy (XPS). A K-Alpha spectrometer (Thermo Fisher Scientific, East Grinstead, UK) was used to perform XPS measurements. The samples were analyzed using a micro-focused, monochromated Al K $\alpha$ X-ray source $(400 \mu \mathrm{m}$ spot size). The kinetic energy of the electrons was measured using a $180^{\circ}$ hemispherical energy analyzer operated in the constant analyzer energy mode (CAE) at $50 \mathrm{eV}$ pass energy for elemental spectra. Thermo Avantage software was used to analyze the spectra. The spectra were fitted with one or more Voigt profiles (binding energy uncertainty: $\pm 0.2 \mathrm{eV}$ ). The analyzer transmission function, Scofield sensitivity factors, ${ }^{51}$ and effective attenuation lengths (EALs) for photoelectrons were applied for quantification. EALs were calculated using the standard TPP-2M formalism. ${ }^{52}$ All spectra were referenced to the $\mathrm{C} 1 \mathrm{~s}$ peak of hydrocarbons at $285.0 \mathrm{eV}$ binding energy controlled by means of the well-known photoelectron peaks of metallic $\mathrm{Cu}, \mathrm{Ag}$, and $\mathrm{Au}$.

\section{Results and discussion}

In this study, we report a new approach for the surface functionalization of NCD films via $\mu \mathrm{CS}$ using alkyne-azide cycloaddition click reactions with the purpose of creating small-scale patterns (Fig. 1).

\subsection{Deposition of nanocrystalline diamond films}

The growth of NCD films on silicon wafer substrates was carried out using a plasma-enhanced CVD method. The thickness of the pristine NCD layers was measured by the spectral reflectance method assessing the interference fringes and was found 


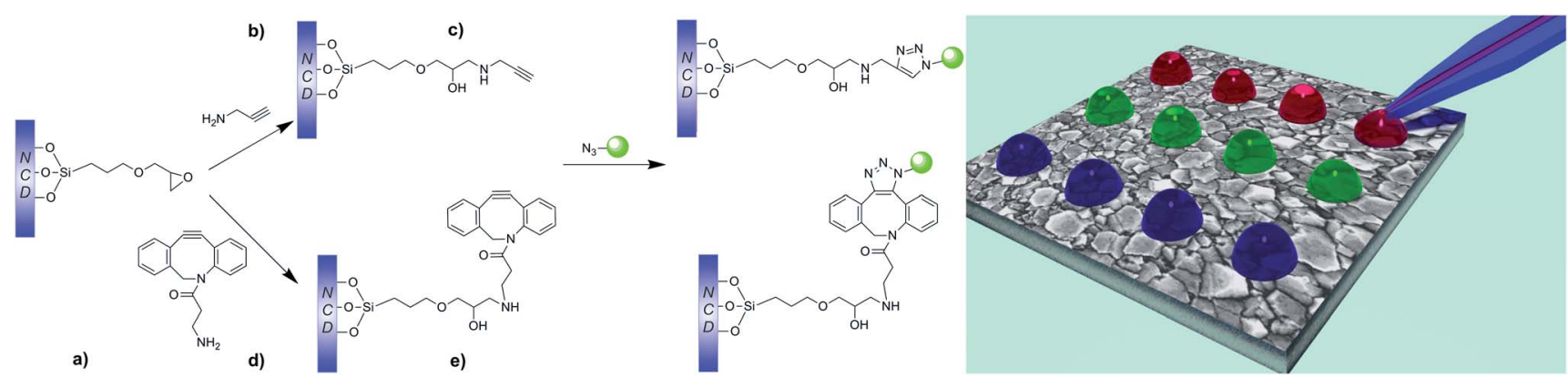

Fig. 1 Schematic representation of the chemical strategy used for the functionalization of NCD films: (a) silanization with (3-glycidyloxypropyl) trimethoxysilane, (b) ring-opening of the epoxide group with propalgylamine to yield an amino alcohol, (c) CuAAC click chemistry reaction of immobilized propargyl groups with incoming azide, (d) ring-opening of the epoxide group with dibenzocyclooctyne-amine (DBCO), and (e) Cufree SPAAC click chemistry reaction of immobilized DBCO groups with incoming azide.

to be $448 \mathrm{~nm}$. Further characterization of the NCD layers was carried out by scanning electron microscopy (SEM), Raman spectroscopy, and atomic force microscopy (AFM). SEM image (Fig. 2a) clearly indicates the polycrystalline nature of a continuous film consisting of grains in sizes up to $200 \mathrm{~nm}$. Moreover, a SEM micrograph of the cross-section of the layer (ESI, Fig. S1 $\dagger$ ) supports the homogeneity of the NCD layer thickness, providing a value of $(435 \pm 13) \mathrm{nm}$, which corresponds well with the measurement by optical means. The topography of the film, as assessed by AFM, also reveals a uniform and homogeneous coverage of the Si substrate with the diamond film (Fig. 2c). The surface roughness $\left(R_{\mathrm{RMS}}\right)$ was evaluated from $5 \times 5 \mu^{2}$ and $1 \times$ $1 \mu \mathrm{m}^{2}$ scanned areas and was determined to be $(16 \pm 2) \mathrm{nm}$. This relatively low value of $R_{\mathrm{RMS}}$ in comparison to other NCD growth methods (typically between 30 and $50 \mathrm{~nm}$ for NCD films) $)^{53}$ is an important feature for many areas of application. It is noteworthy that the roughness can be tuned by varying the deposition parameters such as temperature, gas flow rate, and pressure, as was shown in previous reports. ${ }^{54}$ For instance, it has been reported that the pressure has a sharp influence on the deposition process, as it can lead to inhibition of the film growth. ${ }^{4}$ Increasing the partial pressure of oxygen in the

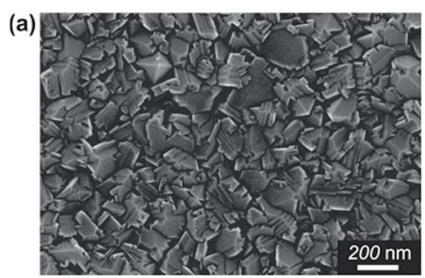

(c)

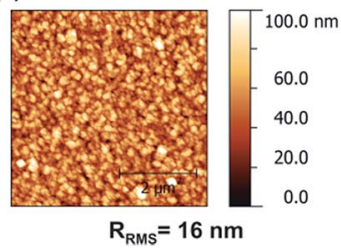

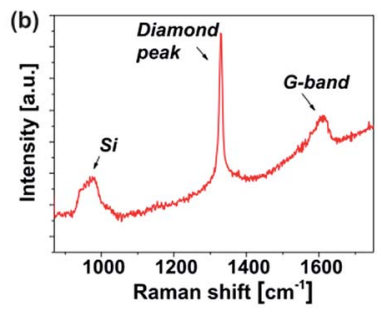

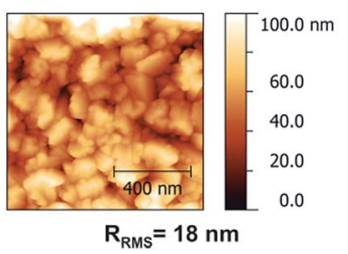

Fig. 2 SEM image of pristine NCD film (a), Raman spectrum of NCD film (b). The sharp peak at $1330 \mathrm{~cm}^{-1}$ proves the diamond character of the deposited films. AFM topography images of NCD film (c). precursor gases either as a pure $\mathrm{O}_{2}$ or in the form of $\mathrm{CO}$ or $\mathrm{CO}_{2}$ has been described for the purpose of enhancing the growth rate and improving the diamond film quality. ${ }^{55,56}$ Moreover, the partial pressure of hydrogen in the feed gas plays an important role in determining the morphology of the film. Its decrease was reported to lead to a change the morphology from discrete wellfaceted diamond grains larger than $100 \mathrm{~nm}$ to finer granular structures with sizes in the order of $30 \mathrm{~nm} .^{57}$

The Raman spectrum (Fig. 2b) is characterized by three strong contributions. The peak centered at $970 \mathrm{~cm}^{-1}$ (Si-peak) reflects the second-order peak of the $\mathrm{Si}$ substrate. ${ }^{58}$ The Raman peak centered at $1330 \mathrm{~cm}^{-1}$ corresponds to the diamond ( $\mathrm{sp}^{3}$-bonding) component. The broad band at approximately $1610 \mathrm{~cm}^{-1}$ is attributed to the non-diamond phase (G-band), i.e., $\mathrm{sp}^{2}$-bonded carbon atoms. ${ }^{4}$ The high intensity of the diamond peak with respect to the G-band indicates a strong predominance of the diamond phase in the film with respect to the graphite component.

\subsection{Modification of NCD films with reactive coatings for $\mu \mathrm{CS}$}

In order to allow for a rapid and well-defined functionalization of the prepared NCD films using $\mu \mathrm{CS}$, the surfaces were coated with a siloxane layer containing two types of chemical groups able to undergo a click chemistry reaction in the presence of an azide-containing molecule. The versatility of silane chemistry has enabled its utilization for a variety of nanoapplications. These range from the silica coating of iron particles to the surface modification of carbon nanofibers for polymer nanocomposites. ${ }^{59-61}$ The preparation of these clickable layers proceeded in two steps: (1) immobilization of a silane containing an epoxide ring, and (2) nucleophilic epoxide opening with a primary amine bound to the clickable group (Fig. 1). While the silane immobilization step was common for both types of layers prepared, the nature of the clickable group immobilized could be changed simply by selecting an appropriate primary amine.

The successful preparation of the targeted layers was confirmed by means of XPS. Fig. 3 shows the changes recorded in the Si $2 \mathrm{p}$ and $\mathrm{N}$ 1s regions of the spectra after the successive reaction steps. The Si $2 \mathrm{p}$ region in particular confirms the attachment of the siloxane layer by the appearance of two 

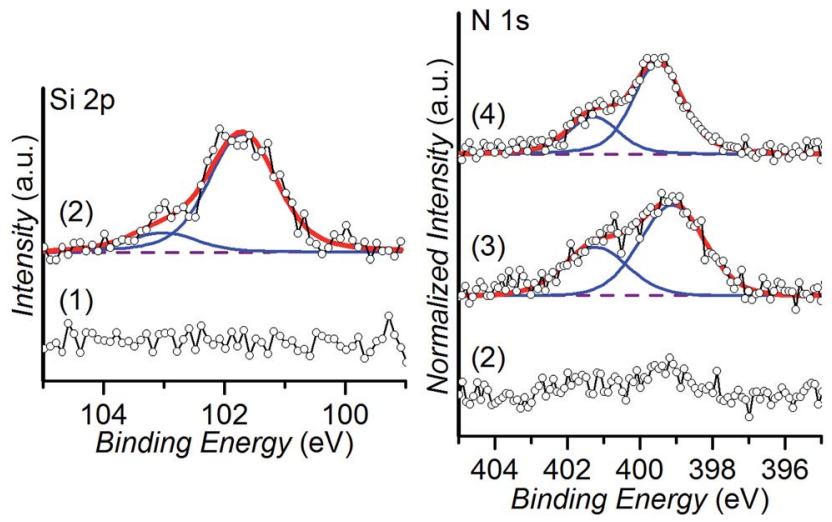

Fig. 3 XPS spectra of the Si $2 p$ (left) and N 1s (right) regions, showing the differences between the bare NCD surface (1), epoxide silane surface (2), propargyl-functionalized NCD (3), and DBCO-functionalized NCD (4).

signals. ${ }^{62}$ They arise from the Si atoms in the layer bonded to different oxygen-containing surface groups and to each other by siloxane bonds. Their binding energies are observed at $101.5 \mathrm{eV}$ and $102.8 \mathrm{eV}$, and indicate that the $\mathrm{Si}$ atoms are bonded to between 1 and 3 oxygen atoms. ${ }^{63,64}$ The signals are broadened by splitting into closely spaced $2 \mathrm{p}_{3 / 2}$ and $2 \mathrm{p}_{1 / 2}$ components due to spin-orbit coupling, which are not individually resolved.

Propargylamine was chosen to prepare the surface to be functionalized using CuAAC. In our previous work we have demonstrated the effectiveness of this strategy for the surface functionalization of model substrates using DPN. ${ }^{65}$ However, in order to overcome the requirement for a transition metal catalyst in the DPN ink formulation, we introduced a surface capable of participating in the SPAAC click reaction. While the CuAAC has enabled great advances in surface functionalization in general, and in particular for DPN and $\mu \mathrm{CS}$, the use of a Cubased catalyst may pose severe limitations for the patterning of some types of biomolecules whose activity may be impaired. Two main strategies have been introduced to increase the reactivity of the alkyne group in order to enable the cycloaddition reaction with an azide to proceed without catalyst, involving the activation of the triple bond with fluorine electron-withdrawing groups or placing it in a strained cycle. ${ }^{66}$ In particular, dibenzocyclooctynes (DBCOs) show significantly increased reaction rates with azides, which allow for the strainpromoted alkyne-azide cycloaddition to proceed in absence of any $\mathrm{Cu}$-species. ${ }^{67}$ Thus, a DBCO-presenting a primary amine group was chosen in this work for the nucleophilic attack to the epoxide ring (Fig. 1). The binding of both types of amines to the surface is observed in the XPS spectra of the $\mathrm{N} 1$ s region, where signals are observed corresponding to $\mathrm{C}-\mathrm{N} / \mathrm{O}=\mathrm{C}-\mathrm{N}$ groups $(399.5 \mathrm{eV})$ and to partially protonated amino groups $(401.3 \mathrm{eV}),{ }^{62,68}$ in contrast to the non-reacted epoxide surface (Fig. 3).

\subsection{Pattern fabrication via $\mu \mathrm{CS}$ and comparison of reactions}

The ability to create microarrays on the NCD films modified with "clickable" coatings was demonstrated using $\mu \mathrm{CS}$. This was achieved by spotting the ink with an SPT attached to a DPN platform where the sample stage can be moved with high precision in all three directions $\left(x, y\right.$ and $z$ ). ${ }^{21}$ The spotting process was relatively fast as it can yield an area of $500 \times 500$ $\mu \mathrm{m}^{2}$ with 100 dots in about $2 \mathrm{~min}^{69}$

Firstly, we compared two different types of click chemistry reactions, i.e. CuAAC and Cu-free SPAAC click reactions, by performing $\mu \mathrm{CS}$ with a fluorescent azide-containing molecule on the NCD films modified with either propargyl or DBCO reactive groups. It should be noted that in both cases after the lithography process was completed the samples were incubated in dark, at room temperature, at a relative humidity of $60 \%$ for 1 $h$ and subsequently washed with ethanol, rinsed with deionized water, and dried. Arrays of dots were printed using a TAMRAazide ink solution (containing $10 \mathrm{mM} \mathrm{CuSO}_{4}$ and $20 \mathrm{mM}$ sodium ascorbate) on the propargylamine-modified NCD film. Fig. 4a shows a fluorescence microscopy image of the fabricated TAMRA-azide spot arrays. All printed spots are uniform in intensity (Fig. 4b). The size distribution histogram of the spots in the array is presented in Fig. $4 \mathrm{c}$ and shows calculated mean radius of the spots in the pattern $10.2 \mu \mathrm{m}$.

To demonstrate a catalyst-free click chemistry reaction on the NCD film, a dibenzocyclooctyne-modified NCD substrate was employed for the printing of the ink solution containing TAMRA-azide but neither $\mathrm{CuSO}_{4}$ nor reducing agent. The obtained fluorescence image (Fig. 4d) shows a homogeneous (a)
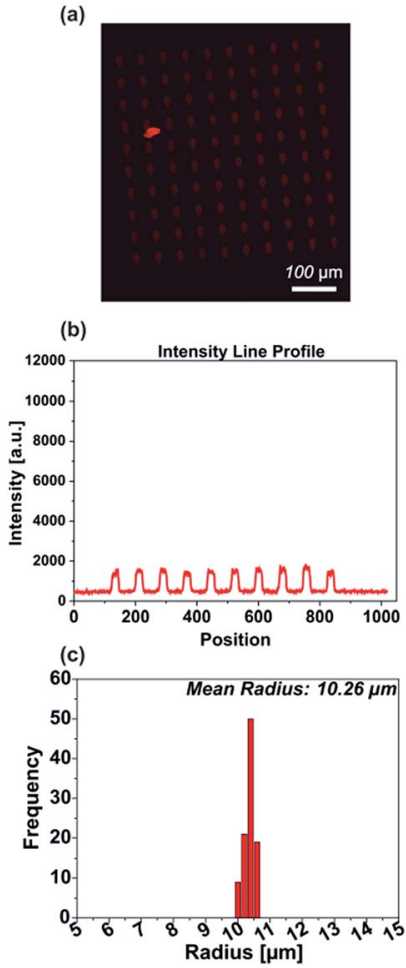

(d)

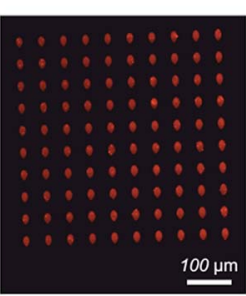

(e)
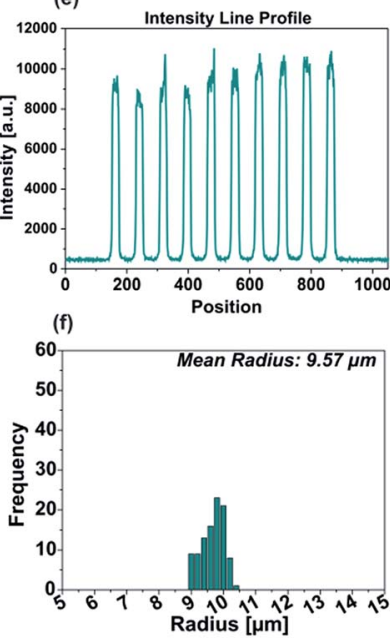

Fig. 4 Fluorescence images of a TAMRA-azide microarray on NCD surfaces modified with propargylamine (a) and dibenzocyclooctyneamine (d). Intensity profiles of line through one row of spots (b and e). Histograms of the spot radius of the images ( $a$ and $d$ ) are given in ( $c$ and f). 
binding of the ink over the whole pattern area after the washing procedure. In contrast to the propargyl-modified NCD surface, a markedly higher fluorescence signal was observed for patterns written on the DBCO-modified NCD surfaces, i.e. where the SPAAC click reaction took place. Furthermore, the intensity profile across one line of spots exhibits a uniform distribution and the average spot radius was $9.5 \mu \mathrm{m}$ (Fig. $4 \mathrm{e}$ and f). In both cases, a narrow distribution of the spot sizes is observed. The significantly increased fluorescence observed on the DBCOmodified NCD surfaces after $\mu C S$ with the TAMRA-azide solution with respect to the propargyl-functional NCD surface indicates that the SPAAC reaction proceeds more rapidly and effectively in comparison with the established CuAAC. Thus, SPAAC could be used to achieve the effective functionalization of the surface after $1 \mathrm{~h}$ of reaction following the printing procedure.

\subsection{Multicomponent arrays}

Multicomponent patterns are a valuable tool as they demonstrate the potential use of the protocol for creating microarrays, important for various types of sensing devices. ${ }^{9}$ In previous works, we have applied several scanning-probe-based patterning techniques in combination with CuAAC for the fabrication of such arrays. ${ }^{24}$

Due to the interest in eliminating the need for the $\mathrm{Cu}$ catalyst, in the current work we fabricated multicomponent arrays employing DBCO-modified NCD substrates. This approach enabled us to assess the effectiveness of the SPAAC reaction for such purpose. For the formation of multicomponent patterns the SPT was first loaded with TAMRA-azide ink and the first sub-pattern of 50 spots was written. Subsequently, the same procedure was applied for the inks Alexa Fluor 488azide and Cy5-azide, respectively. Fig. 5a shows the results of fluorescence measurement after the binding and washing steps. The image evidences the formation of well-resolved subpatterns from the three different fluorescent dyes. Fig. 5b shows a typical fluorescence intensity profile measured along a horizontal line in the multi-component array. It can be seen that while TAMRA-azide shows a stronger signal than Alexa Fluor 455-azide or Cy5-azide, the intensities corresponding to each sub-pattern are highly reproducible. Thus, the possibility to encode the NCD surface with multiple inks containing azidefunctional molecules in a rapid catalyst-free approach is
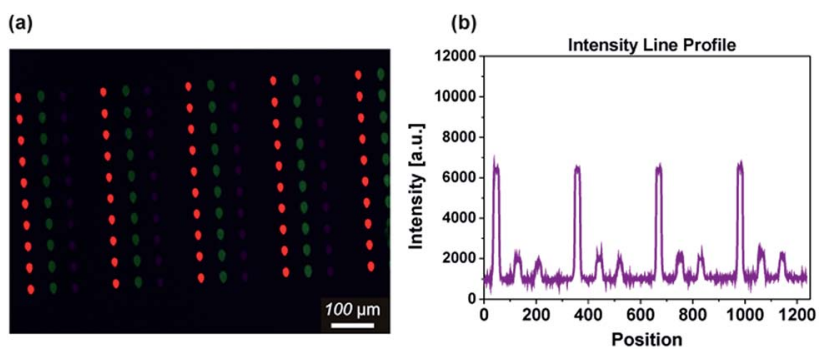

Fig. 5 Fluorescence image of a three spot array (TAMRA azide, Alexa Fluor 488 azide, Cy5-azide) on DBCO-modified NCD surface (a). Intensity profile of line through one row of spots (b). demonstrated. This is of high relevance for the fabrication of biochips, which can exploit the properties of the NCD film.

Arguably, a possible limitation of printing techniques based on scanning probe tools is their low throughput. Importantly, in the case of DPN and related methods, this problem can be easily overcome by parallelization of the process. ${ }^{\mathbf{7 0 , 7 1}}$ This is achieved by fixing several scanning probe tools in parallel while the sample stage is scanned. Thus, the same pattern can be printed many times simultaneously significantly increasing the throughput, which is of relevance for translation of such methods to practical applications.

\section{Conclusions}

A new route for the covalent surface functionalization of nanocrystalline diamond films via catalyst-free click chemistry utilizing spotting with microchannel cantilevers ( $\mu \mathrm{CS}$ ) was introduced. This could be achieved by following a very simple surface modification protocol applied to the NCD film. Moreover, we demonstrated the ability to perform multicomponent patterns using inks containing three different azide-functional molecules and applying them onto the dibenzocyclooctynemodified NCD surface. Results presented herein indicate that formation of a multicomponent micropattern on the modified NCD surface by $\mu$ CS represents a promising strategy for the fabrication of high-density arrays that will be useful in fields such as gas sensors, biosensors, as well as optical devices.

\section{Acknowledgements}

M. D. gratefully acknowledges the support from the Czech Science Foundation via grant 14-06054P. This work was partly carried out with the support of Karlsruhe Nano Micro Facility (KNMF, http://www.kmf.kit.edu), a Helmholtz Research Infrastructure at Karlsruhe Institute of Technology (KIT, http:// www.kit.edu), and the Center for Polymer Technology (CPT) at DWI - Leibniz Institute for Interactive Materials.

\section{Notes and references}

1 M. Davydova, A. Kromka, P. Exnar, M. Stuchlik, K. Hruska, M. Vanecek and M. Kalbac, Phys. Status Solidi A, 2009, 206, 2070-2073.

2 A. Denisenko, A. Romanyuk, C. Pietzka, J. Scharpf and E. Kohn, Diamond Relat. Mater., 2010, 19, 423-427.

3 K. Haenen, K. Meykens, M. Nesladek, G. Knuyt, L. M. Stals, T. Teraji, S. Koizumi and E. Gheeraert, Diamond Relat. Mater., 2001, 10, 439-443.

4 A. Kromka, O. Babchenko, T. Izak, K. Hruska and B. Rezek, Vacuum, 2012, 86, 776-779.

5 K. Tsugawa, M. Ishihara, J. Kim, Y. Koga and M. Hasegawa, Phys. Rev. B: Condens. Matter Mater. Phys., 2010, 82, 125460.

6 P. Rath, S. Ummethala, C. Nebel and W. H. P. Pernice, Phys. Status Solidi A, 2015, 212, 2385-2399.

7 M. Davydova, P. Kulha, A. Laposa, K. Hruska, P. Demo and A. Kromka, Beilstein J. Nanotechnol., 2014, 5, 2339-2345. 
8 P. Rath, M. Hirtz, G. Lewes-Malandrakis, D. Brink, C. Nebel and W. H. P. Pernice, Adv. Opt. Mater., 2015, 3, 328-335.

9 C. Hébert, S. Ruffinatto and P. Bergonzo, in Carbon for Sensing Devices, ed. D. Demarchi and A. Tagliaferro, Springer International Publishing, Cham, 2015, pp. 227-267.

10 M. Davydova, M. Stuchlik, B. Rezek, K. Larsson and A. Kromka, Sens. Actuators, B, 2013, 188, 675-680.

11 P. Villalba, M. K. Ram, H. Gomez, A. Kumar, V. Bhethanabotla and A. Kumar, Mater. Sci. Eng., C, 2011, 31, 1115-1120.

12 A. Kromka, M. Davydova, B. Rezek, M. Vanecek, M. Stuchlik, P. Exnar and M. Kalbac, Diamond Relat. Mater., 2010, 19, 196-200.

13 V. Vermeeren, S. Wenmackers, P. Wagner and L. Michiels, Sensors, 2009, 9, 5600-5636.

14 C. Rodriguez-Emmenegger, C. M. Preuss, B. Yameen, O. PopGeorgievski, M. Bachmann, J. O. Mueller, M. Bruns, A. S. Goldmann, M. Bastmeyer and C. Barner-Kowollik, Adv. Mater., 2013, 25, 6123-6127.

15 J. M. Collins, R. T. Lam, Z. Yang, B. Semsarieh, A. B. Smetana and S. Nettikadan, Lab Chip, 2012, 12, 2643-2648.

16 C. C. Wu, D. N. Reinhoudt, C. Otto, V. Subramaniam and A. H. Velders, Small, 2011, 7, 989-1002.

17 L. S. Wong, F. Khan and J. Micklefield, Chem. Rev., 2009, 109, 4025-4053.

18 D. S. Engstrom, B. Porter, M. Pacios and H. Bhaskaran, J. Mater. Res., 2014, 29, 1792-1816.

19 Y. Liu and J. Yu, Microchim. Acta, 2015, 183, 1-19.

20 A. B. Braunschweig, F. Huo and C. A. Mirkin, Nat. Chem., 2009, 1, 353-358.

21 J. Xu, M. Lynch, J. L. Huff, C. Mosher, S. Vengasandra, G. Ding and E. Henderson, Biomed. Microdevices, 2004, 6, 117-123.

22 M. Ghatkesar, H. Garza, F. Heuck and U. Staufer, Micromachines, 2014, 5, 954-1001.

23 L. Huang, A. B. Braunschweig, W. Shim, L. Qin, J. K. Lim, S. J. Hurst, F. Huo, C. Xue, J. W. Jang and C. A. Mirkin, Small, 2010, 6, 1077-1081.

24 M. Hirtz, A. M. Greiner, T. Landmann, M. Bastmeyer and H. Fuchs, Adv. Mater. Interfaces, 2014, 1, 1300129.

25 M. Hirtz, W. Feng, H. Fuchs and P. A. Levkin, Adv. Mater. Interfaces, 2016, 3, DOI: 10.1002/admi.201500469.

26 H. C. Kolb, M. G. Finn and K. B. Sharpless, Angew. Chem., Int. Ed., 2001, 40, 2004-2021.

27 V. V. Rostovtsev, L. G. Green, V. V. Fokin and K. B. Sharpless, Angew. Chem., Int. Ed., 2002, 41, 2596-2599.

28 A. Barras, S. Szunerits, L. Marcon, N. Monfilliette-Dupont and R. Boukherroub, Langmuir, 2010, 26, 13168-13172.

29 T. Meinhardt, D. Lang, H. Dill and A. Krueger, Adv. Funct. Mater., 2011, 21, 494-500.

30 W. S. Yeap, M. S. Murib, W. Cuypers, X. Liu, B. van Grinsven, M. Ameloot, M. Fahlman, P. Wagner, W. Maes and K. Haenen, ChemElectroChem, 2014, 1, 1145-1154.

31 R. E. Ruther, M. L. Rigsby, J. B. Gerken, S. R. Hogendoorn, E. C. Landis, S. S. Stahl and R. J. Hamers, J. Am. Chem. Soc., 2011, 133, 5692-5694.
32 M. R. Das, M. Wang, S. Szunerits, L. Gengembre and R. Boukherroub, Chem. Commun., 2009, 2753-2755.

33 M. Wang, M. R. Das, M. Li, R. Boukherroub and S. Szunerits, J. Phys. Chem. C, 2009, 113, 17082-17086.

34 S. A. Yao, R. E. Ruther, L. Zhang, R. A. Franking, R. J. Hamers and J. F. Berry, J. Am. Chem. Soc., 2012, 134, 15632-15635.

35 J. C. Jewett and C. R. Bertozzi, Chem. Soc. Rev., 2010, 39, 1272-1279.

36 G. S. McCarty, Nano Lett., 2004, 4, 1391-1394.

37 Q. Wang, T. R. Chan, R. Hilgraf, V. V. Fokin, K. B. Sharpless and M. G. Finn, J. Am. Chem. Soc., 2003, 125, 3192-3193.

38 J. Gierlich, G. A. Burley, P. M. Gramlich, D. M. Hammond and T. Carell, Org. Lett., 2006, 8, 3639-3642.

39 W. F. Paxton, J. M. Spruell and J. F. Stoddart, J. Am. Chem. Soc., 2009, 131, 6692-6694.

40 M. F. Debets, S. S. van Berkel, J. Dommerholt, A. T. Dirks, F. P. Rutjes and F. L. van Delft, Acc. Chem. Res., 2011, 44, 805-815.

41 N. J. Agard, J. A. Prescher and C. R. Bertozzi, J. Am. Chem. Soc., 2004, 126, 15046-15047.

42 R. Manova, T. A. van Beek and H. Zuilhof, Angew. Chem., Int. Ed., 2011, 50, 5428-5430.

43 C. F. Wang, E. M. Makila, M. H. Kaasalainen, D. Liu, M. P. Sarparanta, A. J. Airaksinen, J. J. Salonen, J. T. Hirvonen and H. A. Santos, Biomaterials, 2014, 35, 1257-1266.

44 A. Kuzmin, A. Poloukhtine, M. A. Wolfert and V. V. Popik, Bioconjugate Chem., 2010, 21, 2076-2085.

45 S. V. Orski, A. A. Poloukhtine, S. Arumugam, L. Mao, V. V. Popik and J. Locklin, J. Am. Chem. Soc., 2010, 132, 11024-11026.

46 M. A. Wijdeven, C. Nicosia, A. Borrmann, J. Huskens and F. L. van Delft, $R S C A d v ., 2014$, 4, 10549.

47 S. V. Orski, G. R. Sheppard, S. Arumugam, R. M. Arnold, V. V. Popik and J. Locklin, Langmuir, 2012, 28, 14693-14702.

48 Y. Li, M. Giesbers, M. Gerth and H. Zuilhof, Langmuir, 2012, 28, 12509-12517.

49 J. Escorihuela, A. T. M. Marcelis and H. Zuilhof, Adv. Mater. Interfaces, 2015, 2, 1-42.

50 M. Abramoff, P. Magalhaes and S. Ram, Biophotonics International, 2004, 36-42.

51 J. H. Scofield, J. Electron Spectrosc. Relat. Phenom., 1976, 8, 129-137.

52 S. Tanuma, C. J. Powell and D. R. Penn, Surf. Interface Anal., 2005, 37, 1-14.

53 C. E. Nebel, B. Rezek, D. Shin, H. Uetsuka and N. Yang, in Physics and Applications of CVD Diamond, ed. S. Koizumi, C. Nebel and M. Nesladek, Wiley-VCH Verlag GmbH \& Co. KGaA, Weinheim, Germany, 2008, ch. 6, pp. 129-176.

54 G. Cicala, D. Monéger, D. Cornacchia, P. Pesce, V. Magaletti, G. Perna, V. Capozzi and M. Tamborra, Surf. Coat. Technol., 2012, 211, 152-157.

55 J. A. Mucha, D. L. Flamm and D. E. Ibbotson, J. Appl. Phys., 1989, 65, 3448.

56 M. Frenklach and H. Wang, Phys. Rev. B: Condens. Matter Mater. Phys., 1991, 43, 1520-1545. 
57 Š. Potocký, O. Babchenko, K. Hruška and A. Kromka, Phys. Status Solidi B, 2012, 249, 2612-2615.

58 C. Popov, W. Kulisch, S. Bliznakov, G. Ceccone, D. Gilliland, L. Sirghi and F. Rossi, Diamond Relat. Mater., 2008, 17, 12291234.

59 J. Zhu, S. Wei, I. Y. Lee, S. Park, J. Willis, N. Haldolaarachchige, D. P. Young, Z. Luo and Z. Guo, RSC Adv., 2012, 2, 1136-1143.

60 J. Zhu, S. Wei, J. Ryu, M. Budhathoki, G. Liang and Z. Guo, J. Mater. Chem., 2010, 20, 4937.

61 J. Zhu, S. Wei, A. Yadav and Z. Guo, Polymer, 2010, 51, 26432651.

62 R. A. Shircliff, P. Stradins, H. Moutinho, J. Fennell, M. L. Ghirardi, S. W. Cowley, H. M. Branz and I. T. Martin, Langmuir, 2013, 29, 4057-4067.

63 M. R. Alexander, R. D. Short, F. R. Jones, W. Michaeli and C. J. Blomfield, Appl. Surf. Sci., 1999, 137, 179-183.
64 P. M. Dietrich, S. Glamsch, C. Ehlert, A. Lippitz, N. Kulak and W. E. S. Unger, Appl. Surf. Sci., 2016, 363, 406-411.

65 S. Oberhansl, M. Hirtz, A. Lagunas, R. Eritja, E. Martinez, H. Fuchs and J. Samitier, Small, 2012, 8, 541-545.

66 B. Gold, G. B. Dudley and I. V. Alabugin, J. Am. Chem. Soc., 2013, 135, 1558-1569.

67 P. A. Ledin, N. Kolishetti and G. J. Boons, Macromolecules, 2013, 46, 7759-7768.

68 N. Zydziak, C. M. Preuss, V. Winkler, M. Bruns, C. Hubner and C. Barner-Kowollik, Macromol. Rapid Commun., 2013, 34, 672-680.

69 M. Hirtz, M. Lyon, W. Feng, A. E. Holmes, H. Fuchs and P. A. Levkin, Beilstein J. Nanotechnol., 2013, 4, 377-384.

70 K. Salaita, Y. Wang, J. Fragala, R. A. Vega, C. Liu and C. A. Mirkin, Angew. Chem., Int. Ed., 2006, 45, 7220-7223.

71 J. Xu, M. Lynch, S. Nettikadan, C. Mosher, S. Vegasandra and E. Henderson, Sens. Actuators, B, 2006, 113, 1034-1041. 